\title{
Circulating Current Harmonics Suppression for Modular Multilevel Converters Based on Repetitive Control
}

\author{
Binbin $\mathrm{Li}^{\dagger}$, Dandan $\mathrm{Xu}^{*}$, and Dianguo $\mathrm{Xu}^{*}$ \\ $\dagger^{*}$ School of Electrical Engineering and Automation, Harbin Institute of Technology, Harbin, China
}

\begin{abstract}
Modular multilevel converters (MMCs) have emerged as the most promising topology for high and medium voltage applications for the coming years. However, one particular negative characteristic of MMCs is the existence of circulating current, which contains a dc component and a series of low-frequency even-order ac harmonics. If not suppressed, these ac harmonics will distort the arm currents, increase the power loses, and cause higher current stresses on the semiconductor devices. Repetitive control (RC) is well known due to its distinctive capabilities in tracking periodic signals and eliminating periodic errors. In this paper, a novel circulating current control scheme base on RC is proposed to effectively track the dc component and to restrain the low-frequency ac harmonics. The integrating function is inherently embedded in the RC controller. Therefore, the proposed circulating current control only parallels the RC controller with a proportional controller. Thus, conflicts between the RC controller and the traditional proportional integral (PI) controller can be avoided. The design methodologies of the RC controller and a stability analysis are also introduced. The validity of the proposed circulating current control approach has been verified by simulation and experimental results based on a three-phase MMC downscaled prototype.
\end{abstract}

Key words: Circulating current harmonics, Modular multilevel converter (MMC), Repetitive control

\section{INTRODUCTION}

In recent years, the demand for fully controllable voltage source converter (VSC) topologies is continuously increasing in the fields of electricity networks and motor drives. However, in high-voltage and medium-voltage applications, to meet the requirements of higher voltage ratings and higher conversion efficiency, traditional two-level VSC topologies are always replaced by multilevel converters, such as the diode-clamped multilevel converter, the flying-capacitor converter $(\mathrm{FC})$, the cascaded H-bridge converter (CHB), and the modular multilevel converter (MMC) [1], [2]. Among them, the MMC is the most promising topology since it shows outstanding features such as lower power losses, reduced EMI noise, less semiconductor stresses, scalability, easy assembling, and nearly ideal sinusoidal shaped output waveforms. These

Manuscript received May 30, 2014; accepted Oct. 1, 2014

Recommended for publication by Associate Editor Seung-Ki Sul.

${ }^{\dagger}$ Corresponding Author: libinbinhit@126.com

Tel: +86-451-86418555, Fax: +86-451-86418555, Harbin Inst. of Tech.

* School of Electrical Engineering and Automation, Harbin Institute of Technology, China advantages make the MMC very attractive for high-voltage applications, particularly in the HVDC sector [3]-[8].

The circuit configuration of a three-phase MMC is shown in Fig. 1. Each of its phases consists of two arms, an upper arm and a lower, which are connected through two buffer inductors. Each arm is formed by a series connection of $N$ nominally identical submodules (SMs) and each SM contains a capacitor and either a half-bridge (HB) circuit or a full-bridge (FB) circuit [9]. Due to the series connection of the upper and lower arm SMs, the MMC shows a particular circulating current which circulates through both the upper and lower arms, and ideally, the circulating current should be a dc current. However, since an ac current flows through the SM capacitors, voltage fluctuations are unavoidable. These fluctuations will further impose on the buffer inductors and result in a series of low-frequency harmonics in the circulating current [10]. It follows that these circulating current harmonics are unwanted and should be attenuated. If not, these harmonics will distort the arm currents, bring additional losses, and cause higher current stresses on the semiconductor devices. 

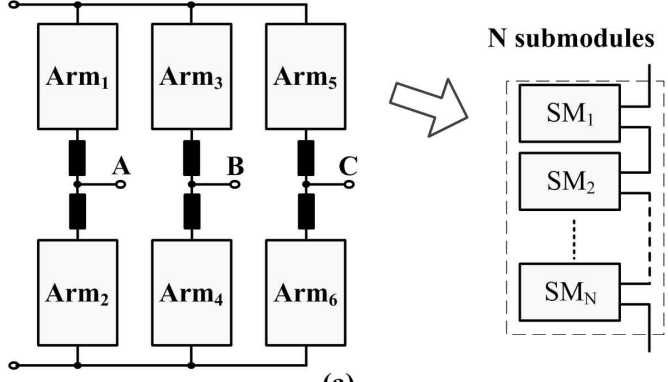



(b) (a)

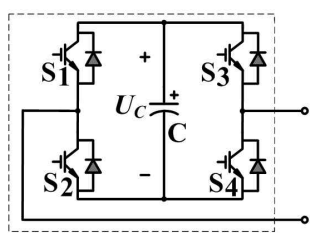

(c)
Fig. 1. Circuit configuration of the modular multilevel converter (MMC). (a) Topology structure. (b) Half-bridge SM. (c) Full-bridge SM.

The circulating current can be controlled by regulating the summation of the upper-arm and the lower-arm voltages [11]. The existing circulating current harmonics suppression methods are generally based on a proportional integral (PI) controller, which can track the dc circulating current reference with no steady-state error [12], [13]. However, this method cannot effectively attenuate the ac harmonics due to its deficient gain at non-zero frequencies. Since the most dominant harmonic frequency in the circulating current is the 2nd order, reference [11] presents another suppression scheme by forming a new PI controller under the second-frequency negative-sequence rotational frame. Nevertheless, this rotated controller needs extra calculation for the coordinate transformations and cannot apply to unbalanced three-phase or single-phase systems. Further, multiple stationary-frame proportional resonant (PR) controllers have been adopted in [14] and [15] by attaching them to a traditional PI controller, and achieving an efficient gain by appropriately setting the resonant frequencies. Although this method is able to suppress the circulating current harmonics accurately, it is too complicated to design a series of multiple PR controllers since the circulating current contains too many harmonics such as the 2nd, 4th and 6th order harmonics.

In addition, circulating current control can also be realized by repetitive control (RC) [16]. RC is based on the internal model principle. This principal encompasses a signal generator for the periodical reference inside the control system to achieve zero steady-state tracking error [17]. The most outstanding merit of RC is its ability to eliminate all of the harmonics in a periodical signal with infinite gains at these harmonic frequencies. In [16], the circulating current control of an MMC is performed by paralleling a traditional PI controller with a RC controller. Thus, the multiple circulating current harmonics can be well attenuated by the RC controller. However, due to the



Fig. 2. Equivalent circuit of one phase of the MMC.

parallel arrangement, RC may conflict with the traditional PI controller. Therefore, it imposes complicated design limitations on the control parameters.

This paper proposes a new circulating current suppression method by only combining an RC controller with a proportional controller. It is demonstrated that the integration function is inherently embedded in the RC controller so that the traditional PI controller can be simplified into a easy proportional controller. Therefore, conflicts between the traditional PI controller and the RC controller can be avoided. The control parameters are easier to design, and the accuracy and robustness of the control system can also be improved. Moreover, the design methodologies of the $\mathrm{RC}$ controller are discussed in terms of the stability and convergence rate. In addition, voltage balancing control of the SM capacitors is also provided. Finally, simulations and experiments are performed to confirm the effectiveness of the proposed control method.

\section{MATHEMATICAL Model OF THE CiRCULATING CURRENT AND REPETITIVE CONTROLLER}

\section{A. Model of the Circulating Current Control in a MMC}

As shown in Fig. 2, an equivalent circuit diagram of one phase of a MMC is used for analysis. $u_{o j}$ is the output voltage of phase $j(j \in\{a, b, c\}), i_{o j}$ is the phase current, and $U_{d c}$ is the dc-link voltage. $u_{u j}, i_{u j}$ and $u_{w j}, i_{w j}$ represent the voltages and currents of the upper arm and lower arm, respectively. The following equations can be obtained by Kirchhoff's voltage and current law:

$$
\begin{gathered}
R i_{u j}+L \frac{d i_{u j}}{d t}=\frac{U_{d c}}{2}-u_{u j}-u_{o j} \\
R i_{w j}+L \frac{d i_{w j}}{d t}=\frac{U_{d c}}{2}-u_{w j}+u_{o j} \\
u_{L j}=R\left(i_{u j}+i_{w j}\right)+L \frac{d\left(i_{u j}+i_{w j}\right)}{d t}=U_{d c}-u_{w j}-u_{u j}
\end{gathered}
$$

where $R$ is the parasitic resistance, and $L$ is the arm inductance. $u_{L j}$ is the voltage across the resistors and inductors, and $i_{c j}$ is the circulating current, which is given as (4). 


$$
i_{c j}=\frac{1}{2}\left(i_{u j}+i_{w j}\right)
$$

Referring to [10], the circulating current always consists of a dc component and multiple even-order harmonics:

$$
i_{c j}=I_{c j, 0}+\sum_{k=2,4,6 \ldots}\left(I_{c j, k} \cos \left(k \omega_{0}+\varphi_{k}\right)\right)
$$

where $I_{c j, 0}$ is the dc component, $I_{c j, k}$ is the amplitude of the $k$-th current harmonic, $\omega_{0}$ is the fundamental angular frequency, $\varphi_{k}$ is the phase angle, and $k$ is an even integer. Specifically, the control objective of the circulating current control is to track the dc component while eliminating the harmonics.

The substitution of (4) into (3) leads to:

$$
2 R i_{c j}+2 L \frac{d i_{c j}}{d t}=U_{d c}-\left(u_{w j}+u_{u j}\right) .
$$

It is seen that the circulating current can be controlled by regulating the summation of the upper-arm and the lower-arm voltages. Further, the transfer function of the circulating current model can be represented as:

$$
G_{p}(s)=\frac{1}{2 L s+2 R} .
$$

\section{B. Repetitive Controller for the Circulating Current Control}

Repetitive controllers (RCs) are widely used to provide precise tracking of periodical and highly complex reference signals. A general block diagram of an RC controller is shown in Fig. 3. The transfer function is:

$$
R C(s)=\frac{U(s)}{E(s)}=\frac{C(s) e^{-s T}}{1-Q(s) e^{-s T}}
$$

where $e^{-s T}$ is a periodic time delay to produce the internal model of an ac signal [17]. $Q(s)$ is a filter to guarantee stability, and $C(s)$ is a compensator to make up the magnitude and phase of the control system.

Since the circulating current of the MMC happens to be a complex signal associated with a series of current harmonics, the RC is a very suitable solution to control them. Applying an RC controller to circulating current control has been discussed in the literature [16]. Note that [16] simultaneously adopts two controllers, an RC controller and a traditional PI controller, in which the PI controller regulates the dc component of the circulating current, and the RC controller is used to attenuate unwanted ac harmonics. However, conflicts are inevitable between these two controllers. This causes complicated limitations in the controller design and degrades the performances of the RC controller.

The repetitive control scheme proposed in [16] is shown in Fig. 4, where a repetitive controller $R C(s)$ is paralleled to a PI controller $P I(s)$. The circulating current $i_{c j}$ was then controlled to track its reference $i_{c j}^{*}$. Thereby, the control plant seen from the repetitive controller $H^{\prime}(s)$, can be derived as:

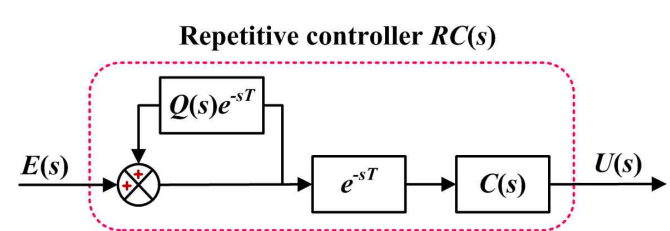

Fig. 3. Block diagram of a general repetitive controller (RC).

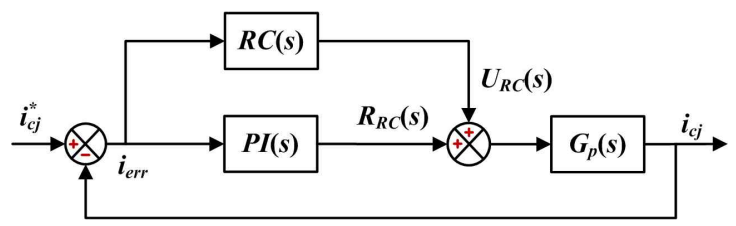

Fig. 4. Control structure in [16].
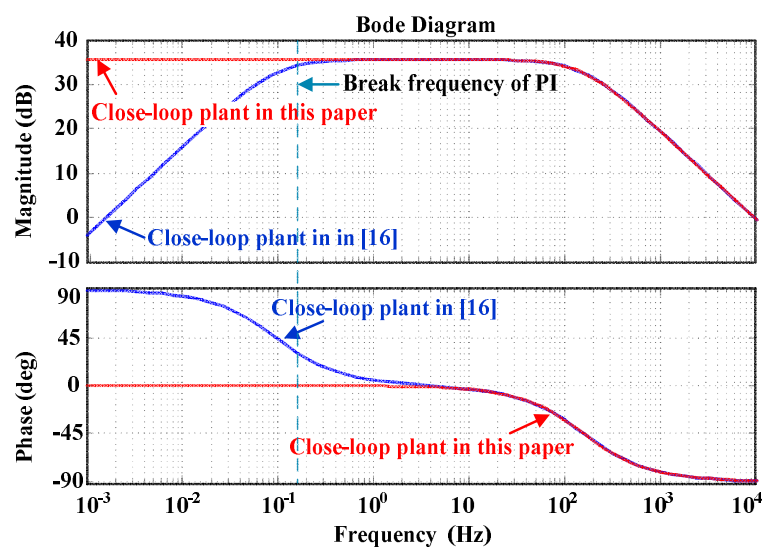

Fig. 5. Different plants of repetitive controllers in [16] and in this paper.

$$
H^{\prime}(s)=\frac{i_{c j}}{U_{R C}(s)}=\frac{G_{p}(s)}{1+G_{p}(s) * P I(s)} .
$$

where $U_{R C}(s)$ denotes the output of the repetitive controller $R C(s)$. Hence, $H^{\prime}(s)$ needs to be carefully considered when designing the repetitive controller. Further, the open-loop transfer function of the current control system can be obtained as:

$$
G(s)=(R C(s)+P I(s)) G_{p}(s)
$$

Fig. 5 and Fig. 6 show a bode diagram of $H^{\prime}(s)$ and $G(s)$, respectively. It should be noted that the gain of $H^{\prime}(s)$ attenuates below the break frequency of $\operatorname{PI}(s) f_{\text {break }}$, while $P I(s)=K_{\mathrm{P}}+K_{\mathrm{I}} / s$ and $f_{\text {break }}=K_{\mathrm{I}} / K_{\mathrm{P}} . H^{\prime}(s)$ should hold high gains at harmonic frequencies for good repetitive control. In addition, it can be seen from Fig. 6 that the harmonics tracking capability of the repetitive control is decreased if $f_{\text {break }}$ is above the $2^{\text {nd }}$ order frequency. Hence, in order to guarantee the validity of repetitive controller in [16], there is a strict restriction on the PI controller so that $f_{\text {break }}$ must be chosen below the $2^{\text {nd }}$ order frequency. Otherwise, conflicts between the RC and PI are inevitable.

\section{Proposed Circulating CURRENT CONTROL METHOD}





Fig. 6. Open-loop bode diagram of current control system with different $f_{\text {break }}$ of PI controller.

This paper adopts a parallel structure, as shown in Fig. 7, for controlling the circulating current. A repetitive controller is paralleled to a proportional controller $P(s)$ instead of a PI controller $P I(s)$. Specifically, the proposed control scheme removes the $f_{\text {break }}$ restriction, which exists in [16]. This is due to the fact that the integrator is inherently incorporated into the repetitive controller. Thus, conflicts are avoided and the designs of the $\mathrm{RC}$ and $\mathrm{P}$ controllers can be mutually independent.

\section{A. Inherent Integration Characteristic of Repetitive Control}

With respect to the general repetitive controller shown in Fig. 3 , and assuming that $Q(s)$ and $C(s)$ are both constant $1 \mathrm{~s}$, the ideal $R C(s)$ can be written as:

$$
R C(s)=\frac{U(s)}{E(s)}=\frac{e^{-s T}}{1-e^{-s T}}=-\frac{1}{2}+\frac{1}{2}\left(\frac{1+e^{-s T}}{1-e^{-s T}}\right)
$$

where $\omega=2 \pi / T$. The following mathematical derivation can be obtained as:

$$
\begin{aligned}
\frac{1+e^{-s T}}{1-e^{-s T}} & =\frac{e^{s T / 2}+e^{-s T / 2}}{e^{s T / 2}-e^{-s T / 2}}=\frac{e^{\pi(s / \omega)}+e^{-\pi(s / \omega)}}{e^{\pi(s / \omega)}-e^{-\pi(s / \omega)}} \\
& =\frac{s / \omega}{\pi} \sum_{k=-\infty}^{\infty} \frac{1}{(s / \omega)^{2}+k^{2}} \\
& =\frac{\omega}{\pi}\left(\frac{1}{s}+\sum_{k=1}^{\infty} \frac{2 s}{s^{2}+(k \omega)^{2}}\right)
\end{aligned}
$$

where $\mathrm{k}$ is integer.

Substituting (12) into (11) results in:

$$
R C(s)=-\frac{1}{2}+\frac{\omega}{2 \pi}\left[\frac{1}{s}+\sum_{k=1}^{\infty} \frac{2 s}{s^{2}+(k \omega)^{2}}\right]
$$

Therefore, it is shown that $R C(s)$ inherently consists of an integrator and a series of resonant controllers. Thus, the control gains at the resonant frequencies as well as the dc frequency are theoretically infinite. It is worth noting that the $R C(s)$ intrinsically contains the integration function.



Fig. 7. Structure of the proposed circulating current control scheme.

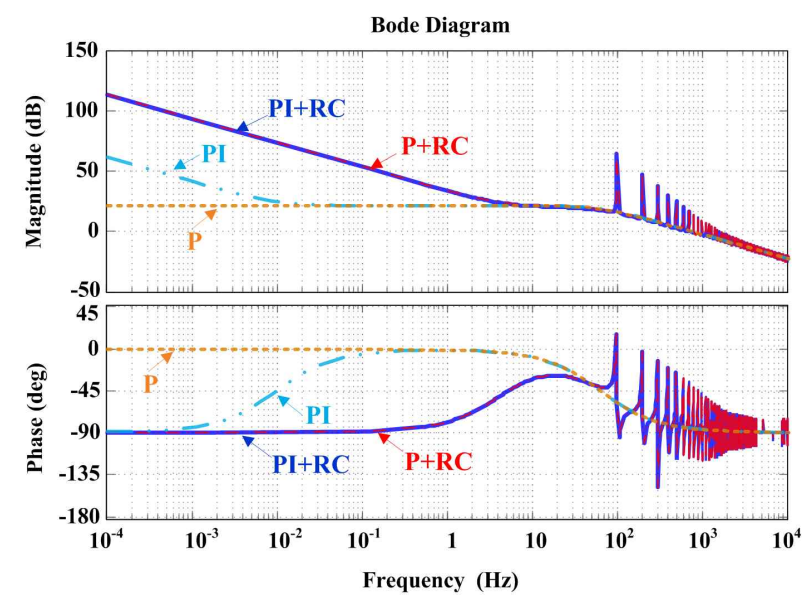

Fig. 8. Comparison of system open-loop bode diagrams with $\mathrm{P}$, $\mathrm{PI},(\mathrm{PI}+\mathrm{RC})$, and $(\mathrm{P}+\mathrm{RC})$.

Therefore, the integrator can be removed and the PI controller can be replaced by a simple $\mathrm{P}$ controller. The control plant seen from the repetitive controller now is improved as:

$$
H(s)=\frac{R_{R C}(s)}{U_{R C}(s)}=\frac{G_{p}(s)}{1+G_{p}(s) * P(s)} .
$$

A bode diagram of $H(s)$ is shown in Fig. 5. This indicates that the break point of the traditional PI controller is removed. Thus, $H(s)$ can ensure a high gain at dc and harmonics frequencies. This is suitable for repetitive controllers.

Moreover, open-loop bode diagrams of the circulating current control systems with different controllers are compared in Fig. 8. It can be observed that the $\mathrm{P}+\mathrm{RC}$ structure is identical to the PI+RC structure (with same magnitude and phase). This shows that the integrator can be saved.

Furthermore, when compared to a traditional PI controller, the presented control scheme can achieve high gains at a series of resonant frequencies to effectively attenuate the ac harmonics of the circulating current.

\section{B. Design of Repetitive Controller}

The design of the repetitive controller concentrates on the following considerations: improving both the stability of the circulating current control system and the response rate of the repetitive controller. The transfer function of the repetitive controller in the discrete domain is given as:

$$
R C(z)=\frac{C(z) * z^{-N_{R C}}}{1-Q(z) * z^{-N_{R C}}}
$$

where $f_{\text {sample }}$ is the sampling frequency, $f_{0}$ is the fundamental frequency (i.e., $50 \mathrm{~Hz}$ ), and $N_{R C}=\left(f_{\text {sample }} /\left(2 f_{0}\right)\right)$ is the ratio of the 
sampling frequency to the $2^{\text {nd }}$ order harmonic frequency.

Further, the transfer function from the error $i_{\text {err }}$ to the circulating current reference $i_{c j}^{*}$ in Fig. 7 can be derived as:

$$
\begin{aligned}
\frac{i_{\text {err }}}{i_{c j}^{*}} & =\frac{1}{1+[P(z)+R C(z)] G_{p}(z)} \\
& =\frac{H(z)}{G_{p}(z)} \times \frac{1-Q(z) z^{-N_{R C}}}{1-[Q(z)-C(z) H(z)] z^{-N_{R C}}} .
\end{aligned}
$$

The characteristic equation of the circulating current control system is:

$$
1-[Q(z)-C(z) H(z)] \times z^{-N_{R C}}=0 .
$$

A necessary condition for system stability is that all of the $N_{R C}$ characteristic roots of (17) are inside the unit circle of the z-plane. Thus, the following constraint must be satisfied to guarantee stability:

$$
D=|Q(z)-C(z) H(z)|<1 .
$$

In order to ensure the stability of the circulating current control system, $Q(z)$ and $C(z)$ should be designed appropriately to satisfy (18). When $Q(z)=1$, the RC controller (15) can be rewritten as:

$$
R C(z)=\frac{z^{-N_{R C}}}{1-z^{-N_{R C}}} .
$$

A pole-zero map of (19) is shown in Fig. 9. The poles, namely the roots of $R C(z)$ all seat at the unit circle. Hence, it is marginally stable for $R C(z)$, which threatens the stability of the circulating current control system. On the other hand, Fig. 9(a) shows that when $Q(z)$ is selected as 0.95 , all of the poles move inside the unit circle, whereas it impairs the control gain of $R C(z)$ at low-frequency harmonics. Thereby, the desired $R C(z)$ for harmonic suppression is that the low-frequency poles (i.e., $2^{\text {nd }}, 4^{\text {th }}$, and $6^{\text {th }}$ harmonics) are set at the unit circle to get high control gains, while the high-frequency poles should be located inside the circle to guarantee stability. Therefore, this paper designs $Q(z)$ as a moving-average filter (MAF) $\left(z^{-1}+2+z\right) / 4$, which has zero phase-shift and attenuates rapidly at high frequencies. A pole-zero map of this $R C(z)$ is included in Fig. 9(b). It is worth noting that the MAF only moves the high frequency poles into the unit circle without affecting the low-frequency poles of $R C(z)$.

Moreover, the compensator $C(z)$ is designed as:

$$
C(z)=K_{R C} * z^{L} .
$$

where $K_{R C}$ is a proportional coefficient and $L$ is a positive integer. $z^{L}$ is a phase-lead element to compensate the computation delay of the DSP in real implementation. The compensated phase angle produced by $z^{L}$ for a signal with a frequency of $f$ is:

$$
\psi_{\text {lead }}=L \frac{f}{0.5 f_{\text {sample }}} .
$$

In addition, $K_{R C}$ determines the control strength of the RC


Fig. 9. Pole-zero maps of RC. (a). Pole-zero map when $Q(\mathrm{z})=0.95$. (b). Pole-zero map when $Q(\mathrm{z})=\left(z^{-1}+2+z\right) / 4$.

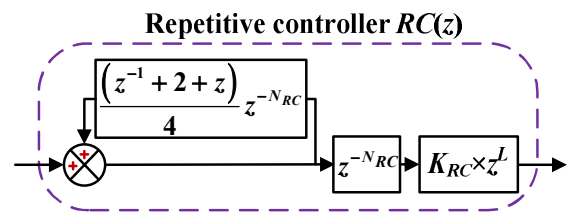

Fig. 10. Designed repetitive controller in discrete domain.

controller, a larger $K_{R C}$ brings a higher convergence rate $D$ and a lower steady-state error. Thus, $K_{R C}$ should be selected so that it is large enough to achieve a high convergence rate. However, the system will be unstable if the oversized $K_{R C}$ leads to $D>1$. Finally, the designed repetitive controller in the discrete domain is shown in Fig. 10.

\section{Complete Control Structure for the MMC}

The complete control strategy for the MMC is depicted in Fig. 11, including the proposed circulating current controller, the overall dc voltage regulation, the SM capacitor voltage balancing control, and the phase-shifted carrier PWM generator. The overall dc voltage regulation ensures that the energy stored in one phase of the MMC remains stabilized by regulating the dc component of the circulating current. The dc-bus voltage $U_{d c}$, is used as a command and compared with one half of the sum of the SM capacitor voltages in phase $j$, that is:

$$
U_{\text {sum_cap } \__{-} j}=\frac{1}{2} \sum_{i=1}^{2 N} U_{\text {cap }_{-} j}(i)
$$

where $U_{\text {cap } j}(i)$ is the capacitor voltage of the $i$-th SM $(i=1 \sim 2 N)$ in phase $j$. A PI controller is then adopted to mitigate this voltage difference and to generate the reference of the dc circulating current $I_{c j, 0}^{*}$, that is:

$$
I_{c j, 0}^{*}=K_{p 1}\left(U_{d c}-U_{\text {sum_cap_ } j}\right)+K_{i 1} \int\left(U_{d c}-U_{\text {sum_cap } \_j}\right) d t
$$

where $K_{p 1}$ and $K_{i 1}$ are the control parameters.

Then, the proposed circulating current controller forces the circulating current to follow $I_{c j, 0}^{*}$, while it attenuates the unwanted harmonics. $K_{p 2}$ is the gain of the proportional converter. Thus, the references for the upper arm and the lower 


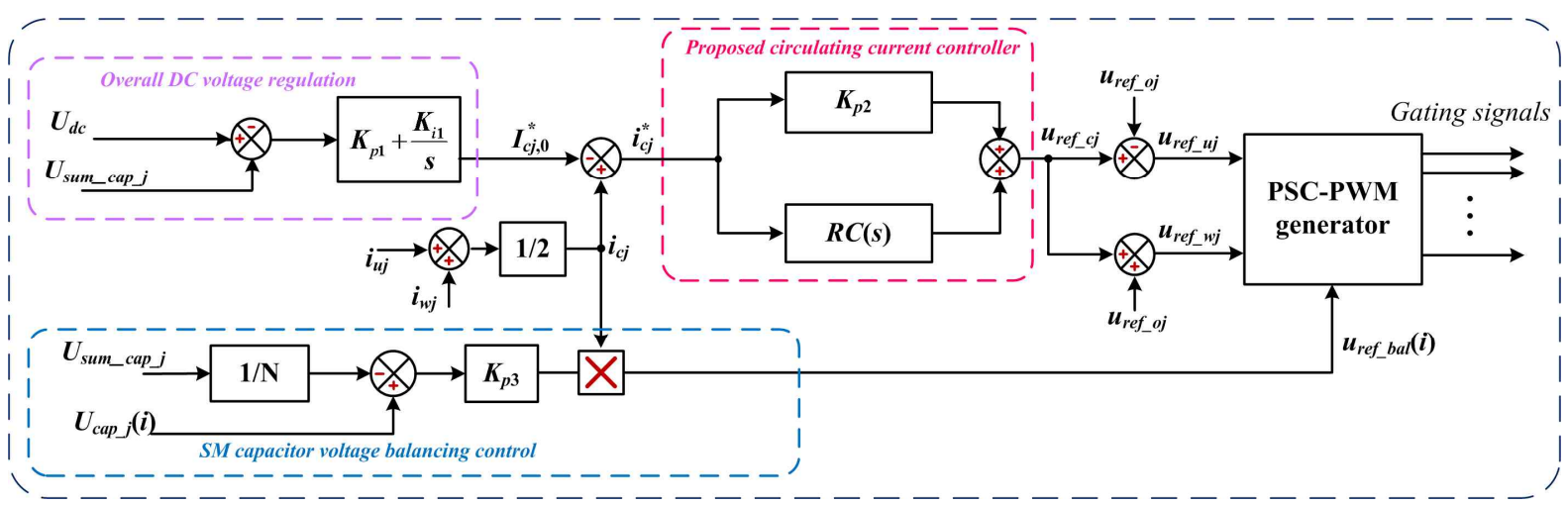

Fig. 11. Proposed current control structure for MMC.

TABLE I

PARAMETERS FOR SIMULATION

\begin{tabular}{cc}
\hline Quantity & Value \\
\hline Number of SMs per arm & $N=3$ \\
DC-bus voltage & $U_{d c}=300 \mathrm{~V}$ \\
Active power & $P=2.5 \mathrm{~kW}$ \\
Reactive power & $Q=0 \mathrm{Var}$ \\
Fundamental frequency & $f_{o}=50 \mathrm{~Hz}$ \\
Rated SM capacitor voltage & $U_{c a p}=100 \mathrm{~V}$ \\
SM capacitance & $C_{s m}=1867 \mu \mathrm{F}$ \\
Buffer inductors & $L=5 \mathrm{mH}$ \\
Arm equivalent resistance & $R=2 \Omega$ \\
Switching frequency & $f_{c}=3 \mathrm{kHz}$ \\
\hline
\end{tabular}

arm voltages can be obtained by combining the output of the circulating current controller $u_{\text {refcir }}$ with the ac voltage reference $u_{\text {ref_oj: }}$ :

$$
\begin{aligned}
& u_{r e f \_u j}=u_{r e f \_c j}-u_{r e f \_o j} \\
& u_{r e f \_w j}=u_{r e f \_c j}+u_{r e f \_o j} .
\end{aligned}
$$

Additionally, from a practical point of view, the MMC SMs can never be identical because parametric differences are unavoidable, such as the component parameter variations, unequal power distribution, and the inconsistent transmission delay of the driving signals. Further, it should be noted that all of these parametric differences will ultimately lead to a capacitor voltage unbalance among the SMs. As shown in Fig. 9, the SM capacitor voltage balancing control is realized by adding an adjustment to the reference signal of each SM, which multiplies the capacitor voltage error with the circulating current, that is:

$$
u_{r e f \_b a l}(i)=K_{p 2}\left(\frac{U_{\text {sum_cap_j }} j}{N}-U_{c a p_{-} j}(i)\right) \times i_{c j}^{e r r}
$$

where $u_{\text {ref bal }}(i)$ represents the balance adjustment of the $i$-th $\mathrm{SM}$, and $K_{p 3}$ denotes the balancing gain. This equation indicates that for SMs with voltages lower than the average voltage, the product of $u_{\text {ref_bal }}(i)$ and the circulating current $i_{c j}$ will form a positive power transfer to charge these SMs. On the other hand, a negative power transfer will be generated to

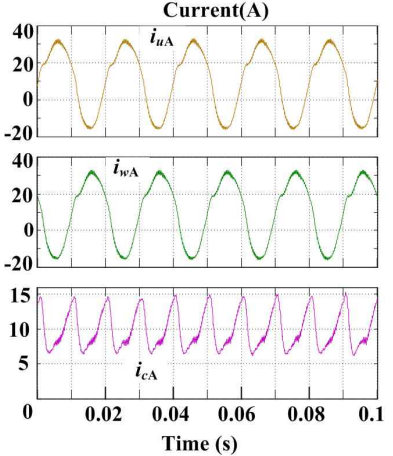

(a)



(b)
Fig. 12. Currents and FFT analysis of phase A in steady-state with PI. (a). Upper arm current $i_{u A}$, lower arm current $i_{w A}$, circulating current $i_{c A}$. (b) FFT analysis of $i_{c A}$.

discharge the SMs when their voltages are higher than the average voltage.

Finally, phase-shifted triangular carriers are adopted for comparison with the references of the SMs, which produces the gating signals for the IGBTs in each of the SMs.

\section{SIMULATION}

A simulation model of a three-phase MMC is established in MATLAB/Simulink software to verify the validity of the proposed repetitive control method. The parameters of the simulation model are listed in Table I. The simulation results under traditional PI control and under the proportional repetitive control are both provided. Fig. 12(a) shows the simulation waveform when the traditional PI controller is adopted. It can be seen that the arm currents are clearly distorted and that significant circulating current harmonics exist. This can be confirmed by the harmonic spectrums in Fig. 12(b). On the other hand, when the designed RC controller is adopted, as can be observed in Fig. 13, almost all of the current harmonics are eliminated, which results in a smooth dc circulating current. Moreover, Fig. 14 indicates that the SM capacitor voltages are well balanced with the proposed SM balancing control method. 


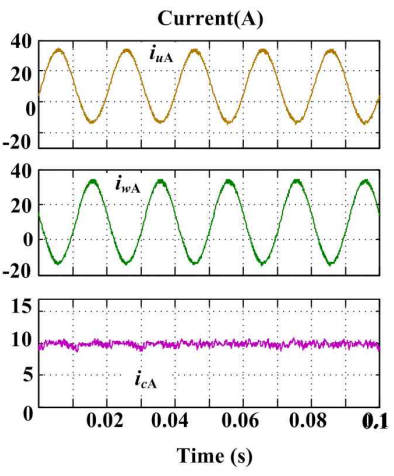

(a)



(b)
Fig. 13. Currents and FFT analysis of phase A in steady-state with RC. (a). Upper arm current $i_{u A}$, lower arm current $i_{w A}$, circulating current $i_{c A}$. (b) FFT analysis of $i_{c A}$.


Fig. 14. Capacitor voltages of upper-arm and lower-arm SMs with RC.
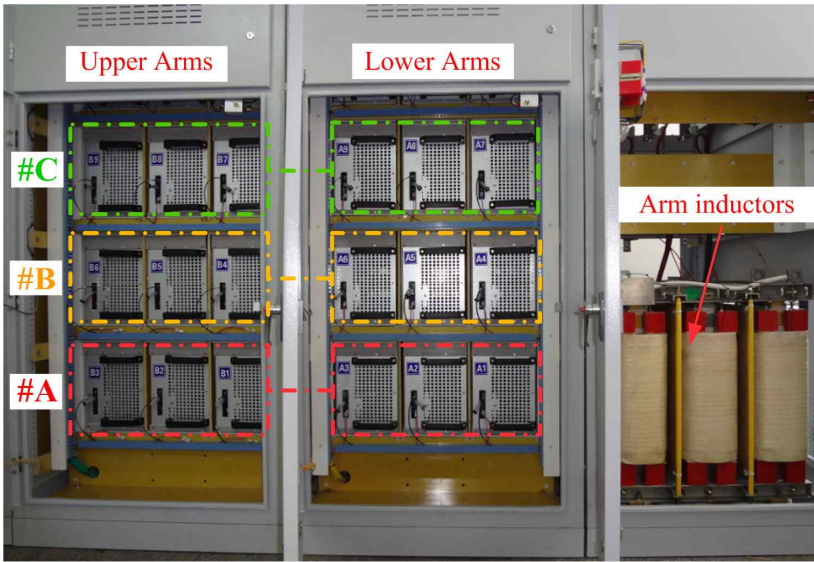

Fig. 15. Photograph of the prototype.

\section{EXPERIMENTAL RESULTS}

To further verify the effectiveness of the proposed circulating current control method, a downscaled three-phase MMC prototype with three SMs per arm has been built in the laboratory, as shown in Fig. 15. The circuit parameters are listed in Table II. The proposed circulating current controller is implemented in a TMS320F28335 floating-point DSP, and an EP3C25Q240C8 FPGA is adopted to perform the PWM modulation and to communicate with all of the SMs. The control parameters for the proposed circulating current controller are designed as follows: the current sample frequency is $f_{\text {sample }}=10 \mathrm{kHz}, N_{R C}=100, K_{R C}=7.8 \mathrm{~V} / \mathrm{A}$, and the
TABLE II

PARAMETERS IN EXPERIMENTS

\begin{tabular}{cc}
\hline Quantity & Value \\
\hline Number of SMs per arm & $N=3$ \\
DC-bus voltage & $U_{d c}=390 \mathrm{~V}$ \\
Active power & $P=2.8 \mathrm{~kW}$ \\
Reactive power & $Q=0 \mathrm{Var}$ \\
Fundamental frequency & $f_{o}=50 \mathrm{~Hz}$ \\
Rated SM capacitor voltage & $U_{c a p}=130 \mathrm{~V}$ \\
SM capacitance & $C_{s m}=1867 \mu \mathrm{F}$ \\
Buffer inductors & $L=5 \mathrm{mH}$ \\
Switching frequency & $f_{c}=3 \mathrm{kHz}$ \\
\hline
\end{tabular}



(a)

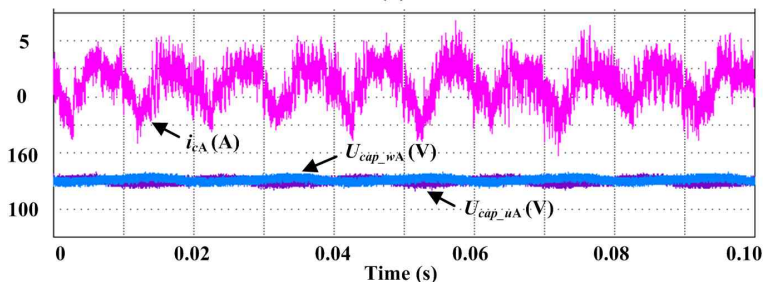

(b)

Fig. 16. Experimental results with traditional PI control method: (a). Currents of upper and lower arms $i_{u A}, i_{w A}$, output current $i_{O A}$. (b). Circlating current $i_{c A}$, capacitor voltage of an upper arm SM $U_{\text {cap_uA }}$, and a lower arm SM $U_{\text {cap_wA }}$.

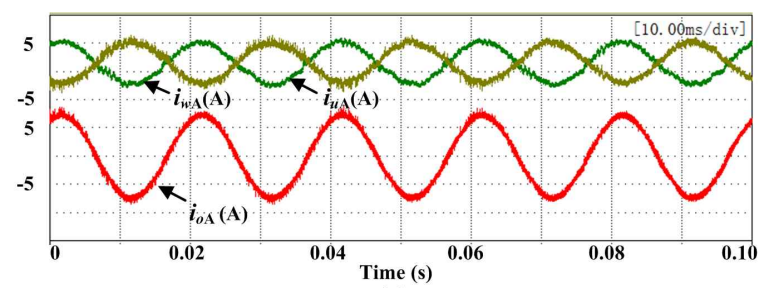

(a)

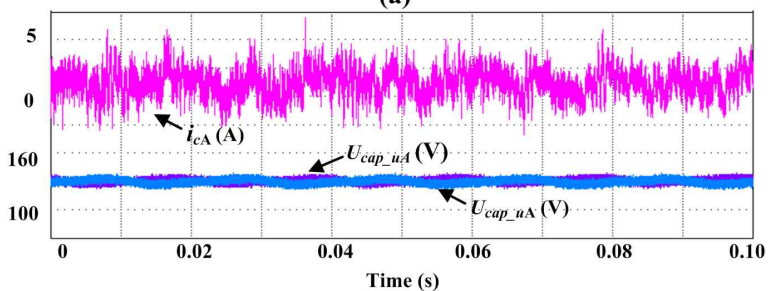

(b)

Fig. 17. Experimental results with proposed circulating current control method: (a). Currents of upper and lower arms $i_{u A}, i_{w A}$, output current $i_{O A}$. (b). Circlating current $i_{c A}$, capacitor voltage of an upper arm SM $U_{\text {cap } \_ \text {A }}$, and a lower arm SM $U_{c a p_{-} w A}$.

proportional gain is $K_{P 2}=31.2 \mathrm{~V} / \mathrm{A}$. Moreover, $L$ is selected as 3 in this experiment. This shows the good compensation of the computation delays. 


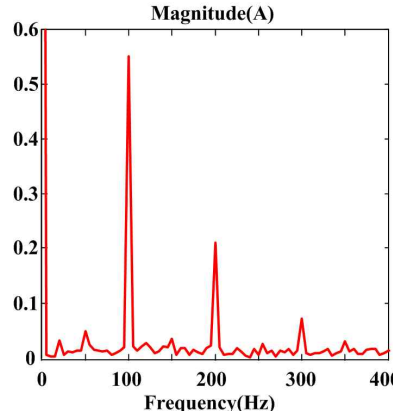

(a)

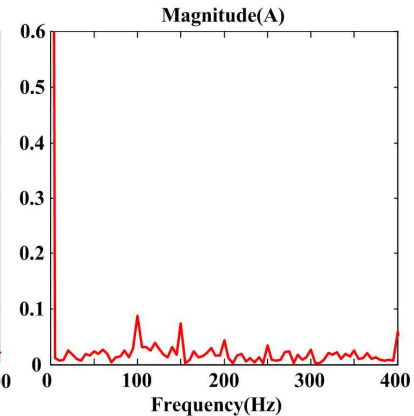

(b)
Fig. 18. FFT anaysis of experimental $i_{c A}$. (a). FFT anaysis of experimental $i_{c A}$ with PI. (b). FFT anaysis of experimental $i_{c A}$ with RC.



(a)

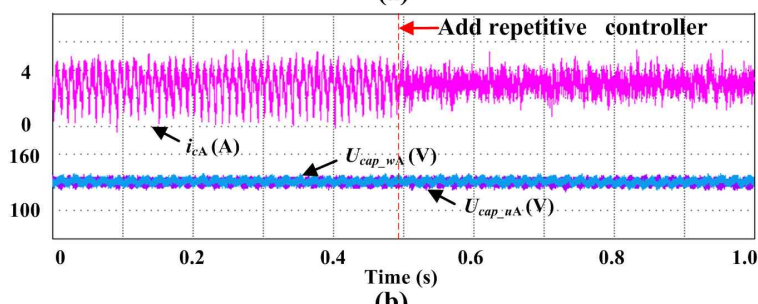

(b)

Fig. 19. Experimental results when proposed circulating current control method is enabel at $\mathrm{t}=0.49 \mathrm{~s}$ : (a). Currents of upper and lower arms $i_{u A}, i_{w A}$, output current $i_{O A}$. (b). Circlating current $i_{c A}$, capacitor voltage of an upper arm SM $U_{c a p \_u A}$, and a lower arm SM $U_{\text {cap } w A}$.

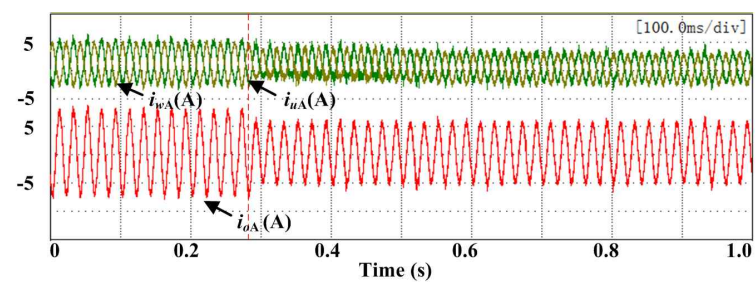

(a)

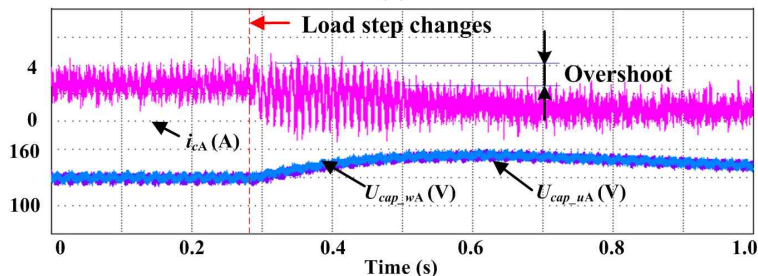

(b)

Fig. 20. Experimental results with the proposed circulating current control method with a load step change at $\mathrm{t}=0.28 \mathrm{~s}$ : (a). Currents of upper and lower arms $i_{u A}, i_{w A}$, output current $i_{o A}$. (b). Circlating current $i_{c A}$, capacitor voltage of an upper arm SM $U_{\text {cap_uA }}$, and a lower arm SM $U_{\text {cap_wA }}$.

The experimental results are shown in Figs. 16-20. The arm currents, output current, circulating current, and capacitor voltages under traditional PI control are shown in Fig. 16. On the other hand, the proposed circulating current control method with the RC is shown in Fig. 17. As further depicted in Fig. 18(a), it is clear that under the traditional PI control method, the $2^{\text {nd }}, 4^{\text {th }}$ and $6^{\text {th }}$ order harmonics are included in the circulating current, due to the limited gain of the PI controller at these ac frequencies. On the other hand, Fig. 18(b) indicates that the magnitudes of the circulating current harmonics are dramatically reduced as a result of the infinite gain of the repetitive controller.

Fig. 19 gives the dynamic performances when the proposed circulating current controller replaces the PI control at $\mathrm{t}=0.49 \mathrm{~s}$. It can be seen that the circulating current harmonics are suppressed quickly after the proposed control is put into action. In the meantime, the capacitor voltages are kept well balanced without any deviations. In addition, the dynamic performances with a load step change are also tested, as shown in Fig. 20. Although overshoot occurs due to the time delay of the repetitive controller, the circulating current gradually converges within about $0.2 \mathrm{~s}$.

\section{CONCLUSIONS}

This paper presents a novel circulating current control method to track the dc component while suppressing the low-frequency ac harmonics. It is shown that the integrating function is inherently integrated in the RC controller, so that only a proportional controller is paralleled with the $\mathrm{RC}$ controller. As a result, conflicts between the RC controller and the traditional PI controller can be avoided, and the RC controller and the proportional controller can be designed independently. The accuracy and robustness of the control system are then improved. Based on the proposed circulating current control method, the complete control structure for the MMC is presented, with emphasis on the SM capacitor voltage balancing. Finally, simulation results are provided and experiments based on a three-phase MMC prototype are performed. Both the simulation and experimental results confirm the validity of the proposed circulating current control method. It can be concluded that the proposed circulating current controller is a flexible and effective control solution for MMCs.

\section{ACKNOWLEDGMENT}

This work was supported by National Natural Science Foundation of China (51237002) and by grants from the Power Electronics Science and Education Development Program of Delta Environmental and Educational Foundation.

\section{REFERENCES}

[1] S. Kouro, M. Malinowski, K. Gopakumar, J. Pou, L. G. 
Franquelo, B.Wu, J. Rodriguez, M. A. Perez, and J. I. Leon, "Recent advances and industrial applications of multilevel converters," IEEE Trans. Ind. Electron., Vol. 57, No. 8, pp. 2553-2580, Aug. 2010.

[2] J. Rodriguez, L. G. Franquelo, S. Kouro, J. I. Leon, R. Portillo, M. M. Prats, and M. A. Perez, "Multilevel converters: An enabling technology for high-power applications," in Proc. IEEE, Vol. 97, No. 11, pp. 1786-1817, Nov. 2009.

[3] K. Shen, J. Wang, D. Zhao, M. Ban, Y. Ji and X. Cai, "Investigation of capacitor voltage regulation in modular multilevel converters with staircase modulation," Journal of Power Electronics, Vol. 14, No. 2, pp. 282-291, Mar. 2014.

[4] R. Marquardt, "Modular multilevel converter: An universal concept for HVDC -Networks and extended DC-Bus-applications," The 2010 International Power Electronics Conference, pp. 502-507, 2012.

[5] S. Allebrod, R. Hamerski, and R. Marquardt, "New transformerless, scalable modular multilevel converters for HVDC-transmission," IEEE Power Electronics Specialists Conference, pp. 174-179, 2008.

[6] N. Ahmed, A. Van, D. Hertem, L. Zhang, and H. -P. Nee, "Prospects and challenges of future HVDC Super Grids with modular multilevel converters," 2011-14th European Conference on Power Electronics and Applications, pp. 1-10, 2011.

[7] J. J. Jung, H. J. Lee, and S. K. Sul, "Control of the modular multilevel converter for variable-speed drives," 2012 International Conference on Power Electronics, pp. $1-6,2012$.

[8] S. Kouro, M. Malinowski, and K. Gopakumar, "Recent advances and industrial applications of multilevel converters," IEEE Trans. Ind. Electron., Vol. 57, No.8, pp. 2553-2580, Aug. 2010.

[9] H. Akagi, "Classification, terminology, and application of the modular multilevel cascade converter (MMCC)," IEEE Trans. Power Electron., Vol. 26, No. 11, pp. 3119-3130, Nov. 2011

[10] K. Ilves, A. Antonopoulos, S. Norrga, and H.-P. Lee "Steady-state analysis of interaction between harmonic components of arm and line quantities of modular multilevel converters," IEEE Trans. Power Electron., Vol. 27, No. 1, pp. 57-68, Jan. 2012.

[11] Q. Tu, Z. Xu, and L. Xu, "Reduced switching-frequency modulation and circulating current suppression for modular multilevel PWM converters," IEEE Trans. Power Del., Vol. 26, No. 3, pp. 2009-2017, Jul. 2011.

[12] M. Hagiwara and H. Akagi, "Control and experiment of pulsewidth-modulated modular multilevel converters," IEEE Trans. Power Electron., Vol. 24, No. 7, pp. 1737-1746, Jul. 2009.

[13] M. Hagiwara, R. Maeda, and H. Akagi, "Control and analysis of the modular multilevel cascade converter based on double-star chopper-cells (MMCC-DSCC)," IEEE Trans. Power Electron., Vol. 26, No. 6, pp. 1649-1658, Jun. 2011.

[14] X. She, A. Huang, X. Ni, and R. Burgos, "AC circulating currents suppression in modular multilevel converter," in Proc. IECON'12, pp. 191-196, 2012.

[15] Z. Li, P. Wang, Z. Chu, H. Zhu, Y. Luo, and Y. Li, “An inner current suppressing method for modular multilevel converters," IEEE Trans. Power Electron., Vol. 28, No. 11, pp. 4873-4879, Nov. 2013.
[16] M. Zhang, L. Huang, W. Yao, and Z. Lu, "Circulating harmonic current elimination of a CPS-PWM-based modular multilevel converter with a plug-In repetitive controller," IEEE Trans. Power Electron., Vol. 29, No. 4, pp. 2083-2097, Apr. 2014.

[17] B. A. Francis and W. M. Wonham, "The internal model principle of control theory," Auto-matica, Vol. 12, No. 5, pp. 457-465, Sep. 1976.

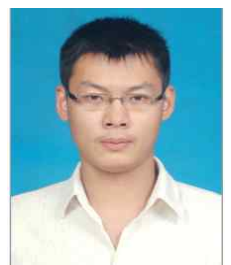

Binbin Li received his B.S. and M.S. degrees in Electrical Engineering from the Harbin Institute of Technology (HIT), Harbin, China, in 2010 and 2012, respectively, where he is currently working toward his Ph.D. degree. His current research interests include high-power electronics, multilevel converters, control algorithms, and PWM techniques.



Dandan Xu was born in Xinjiang, China, in 1990. She received her B.S. degree in Electrical Engineering from the Harbin Institute of Technology (HIT), Harbin, China, in 2013, where she is currently working toward her M.S. degree. Her current research interests include modular multilevel converters.



Dianguo Xu received his B.S. degree in Control Engineering from the Harbin Engineering University, Harbin, China, in 1982, and his M.S. and Ph.D. degrees in Electrical Engineering from the Harbin Institute of Technology (HIT), Harbin, China, in 1984 and 1989 respectively. In 1984, he joined the Department of Electrical Engineering, HIT, as an Assistant Professor. Since 1994, he has been a Professor in the Department of Electrical Engineering, HIT. He was the Dean of the School of Electrical Engineering and Automation, HIT, from 2000 to 2010. He is now the Assistant President of HIT. His current research interests include renewable energy generation technologies, multi-terminal HVDC systems based on VSCs, power quality mitigation, speed sensorless vector controlled motor drives, and high performance PMSM servo systems. He has published over 600 technical papers. Dr. $\mathrm{Xu}$ is a Senior Member of the IEEE, and an Associate Editor for the IEEE Transactions on Industrial Electronics. He is serving as the Chairman of the IEEE Harbin Section, the Director of the Lighting Power Supply Committee of the CPSS, Vice-director of the Electric Automation Committee of the CAA, the Electrical Control System and Equipment Committee of the CES, and the Power Electronics Committee of the CES. 